\title{
The Trochanteric Wing of the Zweymüller Femoral Stem does Not Affect Rotational Stability: An Experimental Study Using a Resin Bone Model
}

\author{
Tomonori Shigemura $^{1}$, Takanori Omae ${ }^{2}$, Shigeru Mitsuhashi ${ }^{3}$, Yasuaki Murata ${ }^{1}$, Yohei Yamamoto ${ }^{1}$, \\ Takashi Sato $^{1}$, Ryuto Tsuchiya ${ }^{1}$, Yuichi Wada ${ }^{1}$, Hiroyuki Takahashi ${ }^{4}$, Michihiro Fujiwara ${ }^{4}$ \\ ${ }^{1}$ Department of Orthopaedic Surgery, Teikyo University Chiba Medical Center, Ichihara City, Japan \\ ${ }^{2}$ Department of Orthopaedic Surgery, Sanmu Medical Center, Sanmu City, Japan \\ ${ }^{3}$ Department of Orthopaedic Surgery, Narashino Dai-ichi Hospital, Narashino City, Japan \\ ${ }^{4}$ Department of Engineering, Teijin Nakashima Medical Co., Ltd, Okayama City, Japan \\ Email address \\ tshigepon@yahoo.co.jp (T. Shigemura)
}

\section{To cite this article:}

Tomonori Shigemura, Takanori Omae, Shigeru Mitsuhashi, Yasuaki Murata, Yohei Yamamoto, Takashi Sato, Ryuto Tsuchiya, Yuichi Wada, Hiroyuki Takahashi, Michihiro Fujiwara. The Trochanteric Wing of the Zweymüller Femoral Stem does Not Affect Rotational Stability: An Experimental Study Using a Resin Bone Model. International Journal of Clinical and Experimental Medical Sciences. Vol. 3, No. 5, 2017, pp. 66-70. doi: 10.11648/j.ijcems.20170305.13

Received: June 28, 2017; Accepted: July 17, 2017; Published: August 9, 2017

\begin{abstract}
The Preserve stem is a modified Zweymüller femoral stem that omits the trochanteric wing. Omission of the trochanteric wing of the prosthesis might influence its rotational stability, but, there are no reports about the rotational stability of a modified Zweymüller femoral stem. The purpose of this study was to investigate the effect of the trochanteric wing on rotational stability by comparing the modified Zweymüller femoral stem and the original Zweymüller femoral stem. Computed tomography of the right femur was used to construct a resin model of the proximal femur. Three types of uncemented stems were used in this study: Preserve $\alpha, \beta$, and exemplary Zweymüller stem. Preserve $\beta$ is a modified Zweymüller-type of stem with a reduced trochanteric wing. Preserve $\alpha$ is also a Zweymüller-type of stem with a further reduced trochanteric wing. The resin bone model was mounted on a universal testing machine. The stem was inserted into the bone model manually and loaded vertically up to $600 \mathrm{~N}$. Then the stem was rotated posteriorly by $1 \% \mathrm{sec}$ until angular rotation was achieved to $5^{\circ}$. The torque at $5^{\circ}$ was compared among the three stems. Vertical displacement of the stem during rotation testing was also recorded as a correlative measure for prosthetic subsidence. The results showed that the mean torque at $5^{\circ}$ was not significantly different among the Preserve $\alpha, \beta$, and exemplary Zweymüller stem (Tukey-Kramer test). Furthermore, the mean vertical displacement amounts during rotation testing were not significantly different among the three stems (Tukey-Kramer test). This study indicate that the Preserve $\alpha$ and $\beta$, modified Zweymüller stems, are rotationally as stable as the exemplary Zweymüller stem.
\end{abstract}

Keywords: Rotational Stability, Zweymüller Stem, Trochanteric Wing

\section{Introduction}

Uncemented femoral stems have beeSn widely used in total hip arthroplasty for osteoarthritis, osteonecrosis, and rheumatoid arthritis or hemiarthroplasty for hip fracture [1-11]. When using uncemented femoral stems, achieving sufficient primary stability is crucial to ensure good long-term results. Lack of primary stability leads to thigh pain and eventually causes loosening or sinking of the prosthesis due to a continuous disruption of bone formation around the implant. Primary stability is ensured by excellent rotational stability. Rotational stability is defined as the implant's mechanical resistance to joint forces that induce rotation around the longitudinal axis of the implant [12].

In 1979, Prof. Dr. Karl Zweymüller introduced a tapered, rectangular, uncemented femoral stem [13]. The design of the stem provides four-point support on the cortical bone in the meta-diaphyseal region of the femur, and the stem broadens in the proximal region to ensure good rotational stability. 
Recently, Kolb A et al. reported that 20-year survival of the Alloclassic Zweymüller stem (Zimmer, Winterthur, Switzerland) was $96 \%$. Nevertheless, the authors stated one disadvantage of the prosthesis was the extensive trochanteric wing of the implant, which should provide stability, but does not allow tissue-sparing surgery [14]. Another problem of implants with trochanteric wing is a thinning of the proximal lateral femur which can result in avulsion fractures of the major trochanter causing abductor weakness and gluteal pain. Therefore, these implants should be adapted for the needs of less invasive surgery.

The Preserve stem (TEIJIN NAKASHIMA MEDICAL CO., LTD., Japan) is a modified implant of the Zweymüller femoral stem. The major change is omission of the trochanteric wing to protect bone of the trochanteric region and avoid trochanteric fractures. Omission of the trochanteric wing of the prosthesis might influence its rotational stability. A Hypothesis that the rotational stability of the Preserve stem was smaller than that of original Zweymüller femoral stem was formed.

Thus, the experimental study was conducted to clarify the effect of the trochanteric wing on rotational stability by comparing the modified Zweymüller femoral stem and the original Zweymüller femoral stem.

\section{Materials and Methods}

\subsection{Preparation}

Computed tomography (CT) of the right femur of a normal 83-year-old man was used to construct a model of the proximal femur. The scan range was $515 \mathrm{~mm}$ and the CT dose index volume was $7.42 \mathrm{mGy}$. Digital imaging data from the scans was then imported into commercial software for medical image processing (Mimics, Materialise, Belgium), in which the bony anatomy was segmented from surrounding structures. Image processing components of the software were used for further refinement of the bony anatomy. The stem was inserted in neutral position, so that the stem axis corresponded with the bone axis of the 3D bone model in the virtual space of the $3 \mathrm{D}$ CAD software SOLIDWORKS (Dassault Systems S. A., France). The data were exported in stereo-lithography (STL) format. This STL file was imported into a multi-material 3D printer (Connex 350, Stratasys, USA) to establish the resin femur model (Figure 1). The area of stem implantation was subtracting from the bone model by using Boolean formula. To fabricate this resin model, hard resin Full Cure 720 (tensile strength 50-65 MPa, Young's modulus 2-3 GPa) was used to replicate cortical bone and soft resin was used to replicate cancellous bone.

Three types of uncemented stems were used in this study: Preserve $\alpha$ (TEIJIN NAKASHIMA MEDICAL CO., LTD., Japan), Preserve $\beta$ (TEIJIN NAKASHIMA MEDICAL CO., LTD., Japan), and exemplary Zweymüller stem. The external appearances of the three stems are shown in Figure 2. Preserve $\beta$ is a modified Zweymüller-type stem with a reduced trochanteric wing compared to the exemplary Zweymüller stem. Preserve $\alpha$ is also a Zweymüller-type stem with a further reduced trochanteric wing.

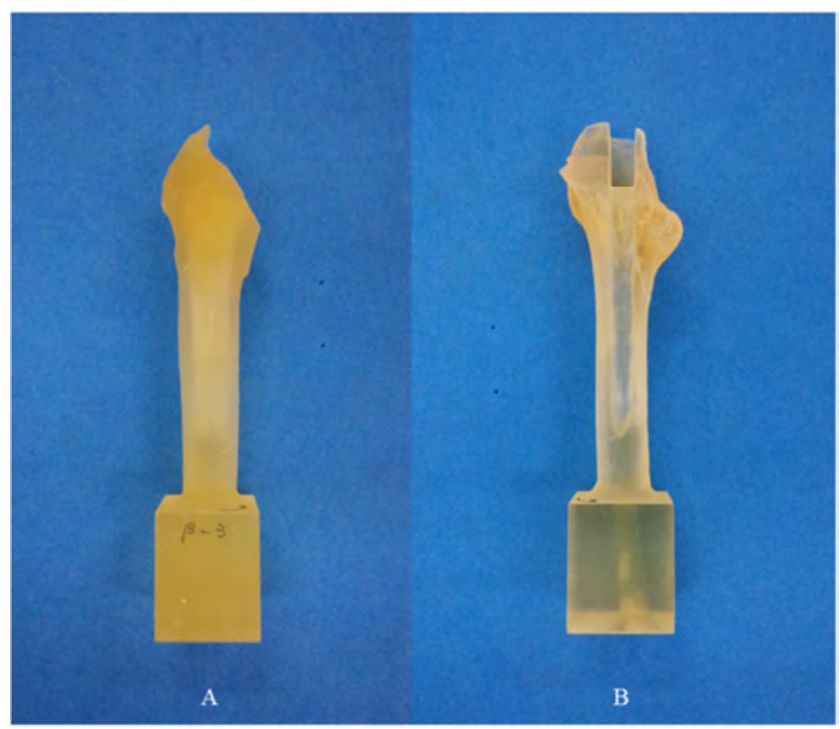

A. frontal view; B. lateral view

Figure 1. Photograph of a resin femur model.

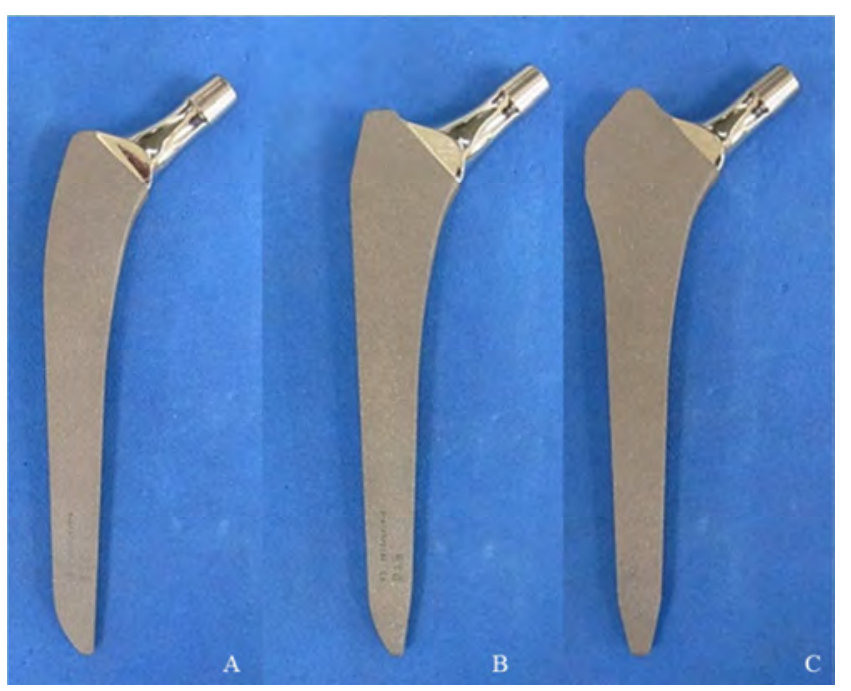

A. Preserve $\alpha$; B. Preserve $\beta$; C. Exemplary Zweymüller stem

Figure 2. Photograph of the four stems.

\subsection{Loading and Testing}

The bone model was mounted on a universal testing machine (Mini Bionix, MTS JAPAN Co., Ltd., Japan) (Figure 3), on which loading and torque could be smoothly changed. The maximum load cell capacity was $25 \mathrm{kN}$. The stem was inserted into the bone model manually and loaded vertically up to $600 \mathrm{~N}(10 \mathrm{~N} / \mathrm{sec})$. Then the stem was rotated posteriorly by $1 \% \mathrm{sec}$ until angular rotation was achieved to $5^{\circ}$, while retaining $600 \mathrm{~N}$ of vertically loading. Torque versus angular rotation (degrees) and the torque at $5^{\circ}$ were recorded and compared among the three stems. Vertical displacement of the stem during rotation testing was also recorded as a correlative measure for prosthetic subsidence. 
Tests were repeated three times for each stem.

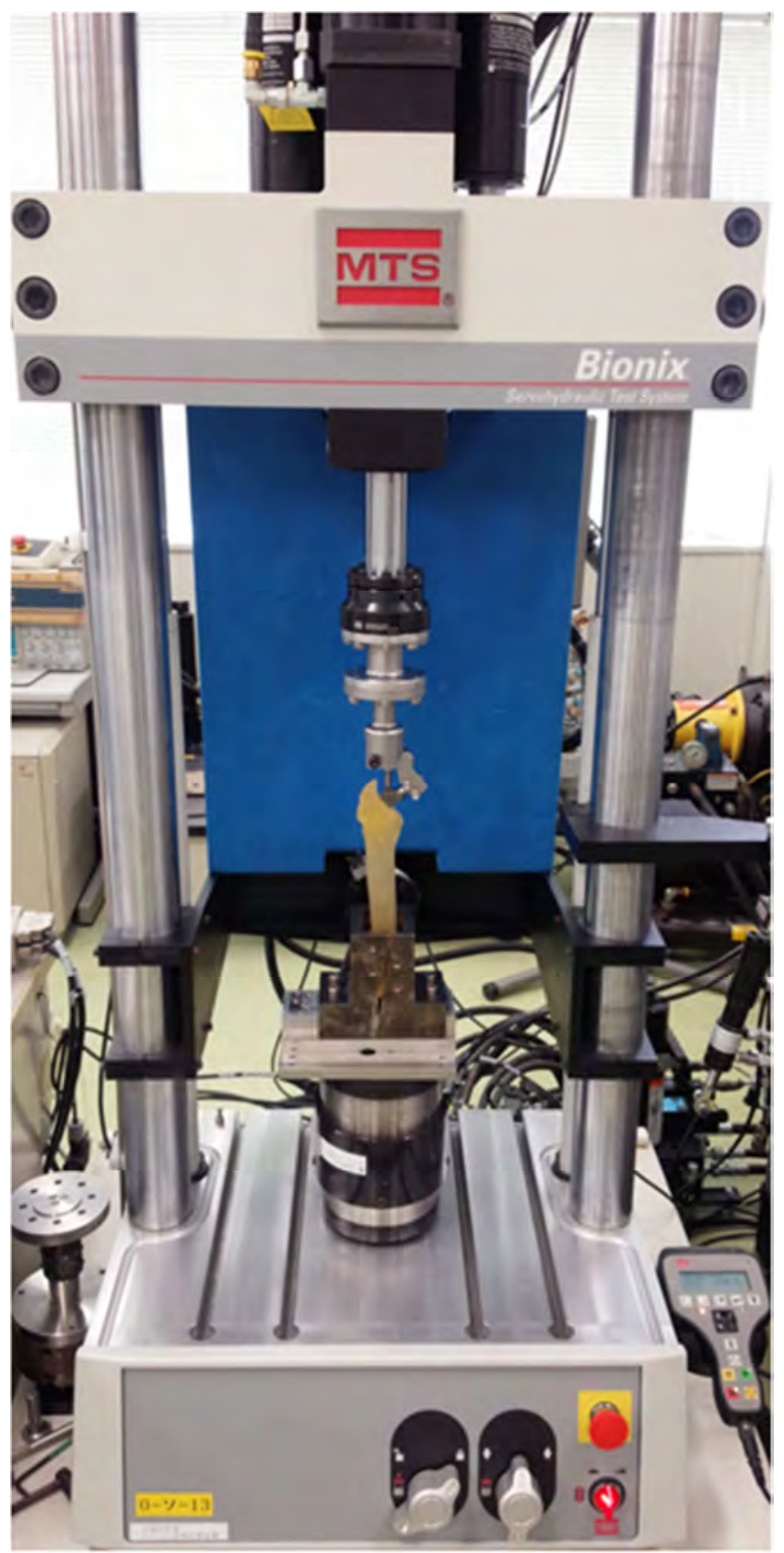

(Mini Bionix, MTS JAPAN Co. Ltd. Japan)

Figure 3. Photograph of the Universal testing machine.

\subsection{Statistical Analysis}

Statistical differences among groups were analyzed with Tukey-Kramer test. Probability values of less than 0.05 were considered significant.

\section{Results}

Figure 4 shows the results of the rotational stability test. There was no significant difference in the mean torque at $5^{\circ}$ among three stems (Tukey-Kramer test).

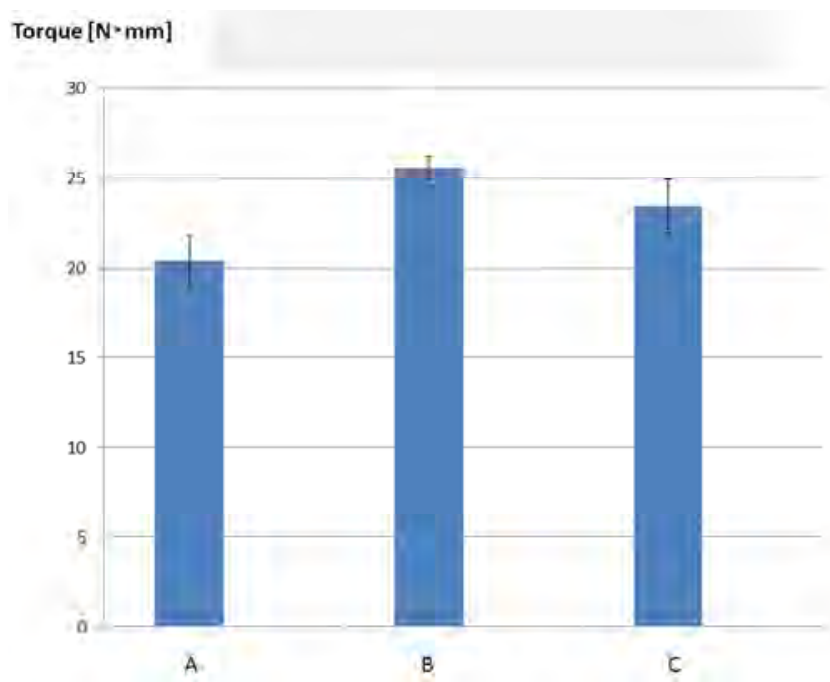

A. Preserve $\alpha$; B. Preserve $\beta$; C. Exemplary Zweymüller stem

Figure 4. Mean torque at 5 degrees.

Furthermore, figure 5 showed that the mean vertical displacement amounts during rotation testing were not significantly different among the three stems (Tukey-Kramer test).

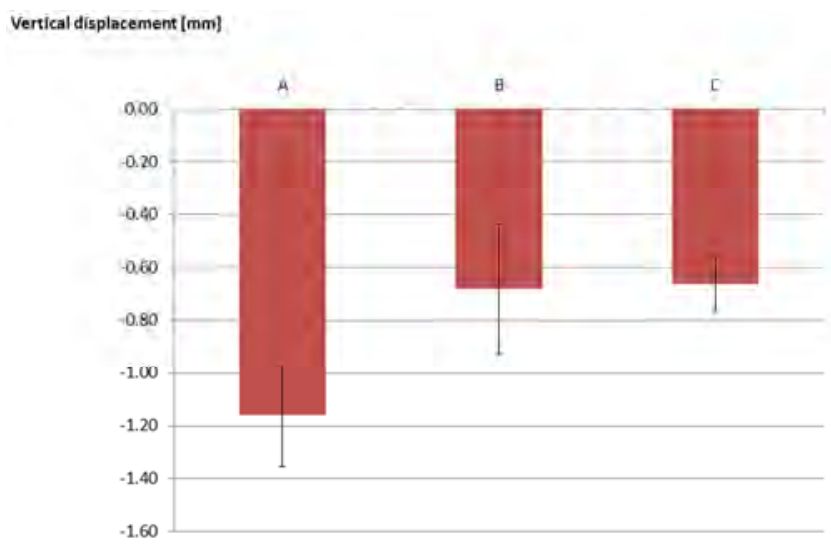

A. Preserve $\alpha$; B. Preserve $\beta$; C. Exemplary Zweymüller stem

Figure 5. Mean vertical displacement.

\section{Discussion}

The proximal region of the Zweymüller stem is important to achieve good rotational stability. If the area of the proximal region is small, a relatively greater surface load is induced, which leads to rotational instability [12]. At first, A hypothesis that the rotational stability of the Preserve stem was smaller than that of original Zweymüller femoral stem was formed. However, the results show that the rotational stability of the Preserve $\alpha, \beta$ and exemplary Zweymüller stem were not significantly different. This result showed that trochanteric wing of Zweymüller stem was not so important for rotational stability. It is possible to speculate because Zweymüller stem are mostly fixed at femoral metaphyseal-diaphyseal junction and it's rectangular cross section provides strong rotational stability with 4 corners embedding into endosteal bone. 
Vertical displacement was not different among the three stems. This result shows that trochanteric wing is not so important to prevent sinking.

Many studies have described good long-term outcomes of Zweymüller femoral stems. Suckel et al. reported a follow-up study of a minimum of 15 years of 320 consecutive Zweymüller total hip arthroplasties [15]. The stem had a survival rate of $98.1 \%$ (308/314) and a Kaplan-Meier estimate of 0.98 (95\% confidence interval, 0.94-0.99) after 17 years. Of 101 patients available for radiographic follow-up, the rate of aseptic loosening was $1.0 \%$ (1/101). Ottink K. et al. also performed survival analysis of 13 to 15 years of follow-up and found the probability of stem survival with revision for aseptic loosening was 0.99 at 10 years and 0.95 after 15 years [16].

In contrast, several studies have reported complications attributed to the trochanteric wing of the Zweymüller stem. Investigators have reported the intraoperative fracture of the trochanteric region occurs between $1.0 \%$ and $5.3 \%$ of the time [17-20]. Kolb A. et al. expressed a preference for the Zweymüller stem because of its excellent primary stability, but also mentioned that the proximal trochanteric wing complicates bone-sparing implantation [14]. Preserve $\alpha$ and $\beta$ reduce the trochanteric wing and this change has the possibility of reduce the risk of intraoperative or postoperative trochanteric fracture. To prove it, further study such as using finite element method is needed.

The present study has several limitations. First, the effect of a single quasi-static torsional load was examined, whereas the forces on the femur in vivo are more varied and repetitive. It is difficult to replicate all in vivo forces in an in vitro experimental setup. Therefore torsional loading was decided to focus on because torsional loading, such as stair climbing, has been shown to cause largest displacements at the bone-implant interface.

Second, a femur model made from resin was used. To the best of knowledge, no study of resin bone models has been reported. The advantages of using a bone model were that the stem could be inserted precisely, replicate exactly the same bone model, and it was cheaper than other methods, such as using cadavers. The disadvantage is that there is no evidence whether this bone model is suitable or not. However, it was able to indicate that the modified Zweymüller stems are as rotationally stable as the original Zweymüller stem relatively.

\section{Conclusions}

The experimental study was conducted to compare the rotational stability of modified Zweymüller stems to an exemplary Zweymüller stem using a resin bone model. Rotational stability of the modified Zweymüller stem was not significantly different from that of an exemplary Zweymüller stem. Furthermore, vertical displacement was not significantly different between modified Zweymüller stems and exemplary Zweymüller stem. The Preserve $\alpha$ and $\beta$, modified Zweymüller stems are rotationally as stable as the exemplary
Zweymüller stem.

\section{References}

[1] Nawaz SZ, Keightley AJ, Desai A, Granville-Chapman J, Elliott D, Newman K, Khaleel A. (2017) Displaced intracapsular neck of femur fractures: Outcome of 810 hydroxyapetite coated (HAC) uncemented hemiarthroplasties. Injury. 48 (4): 909-913.

[2] Mahmoud AN, Kesteris U, Flivik G. (2017) Stable migration pattern of an ultra-short anatomical uncemented hip stem: a prospective study with 2 years radiostereometric analysis follow-up. Hip Int. 27 (3): 259-266.

[3] Koch CN, Mateo LS, Kayiaros S, Cassidy KA, Jerabek SA, Gonzalez Della Valle A. (2016) Spontaneous Fractures of a Modern Modular Uncemented Femoral Stem. HSS J. 12 (3): 250-254.

[4] Murphy CG, Bonnin MP, Desbiolles AH, Carrillon Y, Aït Si Selmi T. (2016) Varus will have varus; a radiological study to assess and predict varus stem placement in uncemented femoral stems. Hip Int. 26 (6): 554-560.

[5] Veen EJ, Schrier JC, Van't Riet E, Breslau MJ, Barnaart AF. (2016) Outcome of the Cementless Zweymüller BICON-PLUS Cup and SL-PLUS Stem in the Very Elderly Individuals. Geriatr Orthop Surg Rehabil. 7 (2): 74-80.

[6] McLaughlin JR, Lee KR. (2016) Long-term results of uncemented total hip arthroplasty with the Taperloc femoral component in patients with Dorr type $\mathrm{C}$ proximal femoral morphology. Bone Joint J. 98-B (5): 595-600.

[7] Inaba Y, Kobayashi N, Oba M, Ike H, Kubota S, Saito T. (2016) Difference in Postoperative Periprosthetic Bone Mineral Density Changes Between 3 Major Designs of Uncemented Stems: A 3-Year Follow-Up Study. J Arthroplasty. 31 (8): 1836-41.

[8] Sueyoshi T, Meding JB, Berend ME, Brunsman MJ, Ritter MA. (2016) Short-term Outcomes with a Second-Generation Uncemented Stem in Total Hip Arthroplasty. Orthopedics. 39 (2): e215-8.

[9] Gastaud O, Cambas PM, Tabutin J. (2016) Femoral revision with a primary cementless stem. Orthop Traumatol Surg Res. 102 (2): 149-53.

[10] Enoksen CH, Gjerdet NR, Klaksvik J, Arthursson AJ, Schnell-Husby O, Wik TS. (2016) Deformation pattern and load transfer of an uncemented femoral stem with modular necks. An experimental study in human cadaver femurs. Clin Biomech (Bristol, Avon). 32: 28-33.

[11] McLaughlin JR, Lee KR. (2016) Total Hip Arthroplasty With an Uncemented Tapered Femoral Component in Patients Younger Than 50 Years of Age: A Minimum 20-Year Follow-Up Study. J Arthroplasty. 31 (6): 1275-8.

[12] Effenberger H, Heiland A, Ramsauer T, Plitz W, Dorn U. (2001) A model for assessing the rotational stability of uncemented femoral implants. Arch Orthop Trauma Surg. 121: p. 60-4.

[13] Zweymüller K, Semlitsch M. (1982) Concept and material properties of a cementless hip prosthesis system with $\mathrm{Al}_{2} \mathrm{O}_{3}$ ceramic ball heads and wrought Ti-6Al-4V stems. Arch Orthop Trauma Surg. 100: p. 229-36. 
[14] Kolb A, Grübl A, Schneckener CD, et al. (2012) Cementless total hip arthroplasty with the rectangular titanium Zweymüller stem: a concise follow-up, at a minimum of twenty years, of previous reports. J Bone Joint Surg Am. 94: p. 1681-4.

[15] Suckel A, Geiger F, Kinzl L, et al. (2009) Long-term results for the uncemented Zweymuller/Alloclassic hip endoprosthesis. A 15-year minimum follow-up of 320 hip operations. J Arthroplasty. 24: p. 846-53.

[16] Ottink K, Barnaart L, Westerbeek R, et al. (2015) Survival, clinical and radiological outcome of the Zweymüller SL/Bicon-Plus total hip arthroplasty: a 15-year follow-up study. Hip Int. 25: p. 204-8.

[17] Garcia-Cimbrelo E, Cruz-Pardos A, Madero R, et al. (2003) Total hip arthroplasty with use of the cementless Zweymüller Alloclassic system. A ten to thirteen-year follow-up study. J Bone Joint Surg Am. 85: p. 296-303.
[18] Delaunay C. (2014) Effect of hydroxyapatite coating on the radio-clinical results of a grit-blasted titanium alloy femoral taper. A case-control study of 198 cementless primary total hip arthroplasty with the Alloclassic ${ }^{\mathrm{TM}}$ system. Orthop Traumatol Surg Res. 100: p. 739-44.

[19] Korovessis P, Repantis T, Zafiropoulos A. (2011) High medium-term survivorship and durability of Zweymüller-Plus total hip arthroplasty. Arch Orthop Trauma Surg. 131: p. 603-11.

[20] Reigstad O, Siewers P, Røkkum M, et al. (2008) Excellent long-term survival of an uncemented press-fit stem and screw cup in young patients: follow-up of 75 hips for 15-18 years. Acta Orthop. 79: p. 194-202. 